\title{
Encapsulated Papillary Thyroid Tumor with Delicate Nuclear Changes and a KRAS Mutation as a Possible Novel Subtype of Borderline Tumor
}

\author{
Kenji Ohba ${ }^{1,2} \cdot$ Norisato Mitsutake ${ }^{3}$ \\ Michiko Matsuse ${ }^{3}$ \\ Tatiana Rogounovitch ${ }^{3}$ \\ Nobuhiko Nishino $^{4}$. Yutaka Oki ${ }^{2}$ \\ Yoshie Goto $^{1}$. Kennichi Kakudo \\ ${ }^{1}$ Department of Internal Medicine, Enshu \\ Hospital, Shizuoka; ${ }^{2}$ Department of Family and \\ Community Medicine, Hamamatsu University \\ School of Medicine, Shizuoka; ${ }^{3}$ Department \\ of Radiation Medical Sciences, Atomic Bomb \\ Disease Institute, Nagasaki University, Nagasaki; \\ ${ }^{4}$ Department of Surgery, Maruyama Hospital, \\ Shizuoka; 'Department of Pathology, Nara \\ Hospital, Kindai University Faculty of Medicine, \\ Nara, Japan
}

Received: November 8, 2018

Revised: December 5, 2018

Accepted: December 7, 2018

\section{Corresponding Author}

Kennichi Kakudo, MD, PhD

Department of Pathology, Nara Hospital, Kindai

University Faculty of Medicine, 1248-1 Otoda-cho,

Ikoma, Nara 630-0293, Japan

Tel: $+81-743-77-0880$

Fax: $+81-743-77-0890$

E-mail: kakudo@thyroid.jp

\begin{abstract}
Although papillary thyroid carcinoma (PTC)-type nuclear changes are the most reliable morphological feature in the diagnosis of PTC, the nuclear assessment used to identify these changes is highly subjective. Here, we report a noninvasive encapsulated thyroid tumor with a papillary growth pattern measuring $23 \mathrm{~mm}$ at its largest diameter with a nuclear score of 2 in a 26-year-old man. After undergoing left lobectomy, the patient was diagnosed with an encapsulated PTC. However, a second opinion consultation suggested an alternative diagnosis of follicular adenoma with papillary hyperplasia. When providing a third opinion, we identified a low MIB-1 labeling index and a heterozygous point mutation in the KRAS gene but not the BRAF gene. We speculated that this case is an example of a novel borderline tumor with a papillary structure. Introduction of the new terminology "noninvasive encapsulated papillary RAS-like thyroid tumor (NEPRAS)" without the word "cancer" might relieve the psychological burden of patients in a way similar to the phrase "noninvasive follicular thyroid neoplasm with papillary-like nuclear features (NIFTP)."
\end{abstract}

Key Words: Borderline/precursor tumor; Observer variation; RAS-like tumor
A subset of thyroid tumors was classified as borderline/precursor tumors in the fourth edition of the World Health Organization (WHO) Classification of Tumors of Endocrine Organs. ${ }^{1-3}$ This categorization was based on evidence from more than 150 publications on noninvasive follicular thyroid neoplasm with papillary-like nuclear features (NIFTP) and accompanied by a discussion of the incidence, diagnosis, and prognostic implications of this terminology in Nikiforov et al. ${ }^{4}$ However, these borderline thyroid tumors were defined only as encapsulated tumors with a follicular growth pattern, a category that included hyalinizing trabecular tumor (HTT), follicular tumor of uncertain malignant potential, well-differentiated tumor of uncertain malignant potential (WDT-UMP), and NIFTP. ${ }^{1-3}$ Because papillary microcarcinoma was proposed to be renamed as papillary microtumor
(PMiT) by thyroid experts, ${ }^{5}$ there would be no reason to restrict borderline/precursor tumors to the follicular growth pattern.

Papillary thyroid carcinoma (PTC)-type nuclear changes are the most reliable morphological features in the diagnosis of PTC. However, the nuclear assessment used to identify these changes is highly subjective. ${ }^{6}$ In particular, the threshold for rendering a diagnosis between follicular adenoma (FA) and encapsulated follicular variant of PTC (FV-PTC) has varied greatly among pathologists. ${ }^{7}$ This issue is not restricted to folliculargrowth patterned tumors. Issues with diagnosis using a nuclear assessment have also been seen in other cases. For example, the main differential diagnosis of encapsulated conventional PTCs is FAs with papillary hyperplasia, ${ }^{8}$ and distinguishing between these two types of tumors poses a challenge similar to that of 
differentiating encapsulated FV-PTCs from FAs.

\section{CASE REPORT}

In September 2016, a 26-year-old man with no past medical history was found to have a nodule at the left lower lobe of the thyroid gland by palpation during an annual medical checkup exam. The patient visited our outpatient clinic at Enshu Hospital for further evaluation. There was no family history of thyroid disorders. Thyroid function tests indicated that the patient had euthyroid status with normal thyroid stimulating hormone (4.18 $\mathrm{mIU} / \mathrm{L}$; reference range, 0.50 to 5.00$)$, FT4 (16.5 nmol/L; reference range, 11.6 to 21.9), and FT3 levels ( $4.7 \mathrm{pmol} / \mathrm{L}$; reference range, 3.5 to 6.6) as measured by Elecsys (Roche Diagnostics K.K., Tokyo, Japan). Serum thyroglobulin was $18.7 \mu \mathrm{g} / \mathrm{L}$ (reference range, $\leq 33.7$ ) and anti-thyroglobulin antibodies were undetectable. B-mode ultrasonography (US) revealed a hypoechoic, heterogeneous nodule $23 \mathrm{~mm}$ at its largest diameter with a slightly jagged border (Fig. 1A). Color Doppler US showed increased intranodular vascularity. Although fine-needle aspiration cytology (FNAC) showed a benign cytological diagnosis, the patient preferred to undergo a left lobectomy of the thyroid rather than continuing with an annual US follow-up. Unexpectedly, the nodule was diagnosed as an encapsulated PTC on histology by a local pathologist. However, when a second opinion was obtained, the nodule was diagnosed as a FA with papillary hyperplasia because of the lack of well-developed PTC-type nuclear features. To determine whether he would benefit from additional resection (completion thyroidectomy), cytological specimens and stored formalin-fixed, paraffin-embedded tissue blocks were analyzed in a third opinion consultation.

FNAC revealed a number of crowded groups of follicular cells with slight nuclear enlargement and mild nuclear overlapping (Fig. 1B). Diagnosis by the doctors providing a third opinion was high-risk indeterminate nodule (follicular neoplasm). The cut surface of the left lobe obtained from hemithyroidectomy demonstrated a well-circumscribed solid tumor. A low-power view showed an encapsulated nodule comprising two types of papillary lesions: one covered with follicular cells containing small hyperchromatic nuclei (adenomatous nodule-like lesion) and the other covered with larger follicular cells having large nonhyperchromatic vesicular nuclei (Fig. 1C, D). Only a trace amount of follicular pattern was noted in this tumor. In highpower view (Fig. 1E, F), the second type of papillary lesion showed nuclear enlargement, irregularity, elongation, and overlapping as well as a few nuclear grooves. Nuclear pseudoinclu- sions were absent and chromatin characteristics were insufficient. As PTC-type nuclear features were equivocal (with a nuclear score of two according to Nikiforov et al., ${ }^{4}$ the doctors providing a third opinion consultation did not endorse the previously suggested diagnoses of encapsulated PTC and FA with papillary hyperplasia. Additionally, these pathological findings did not fulfil the diagnostic criteria for new borderline tumor entities (i.e., NIFTP and WDT-UMP) according to the WHO classification, which not only are intended to be applied to encapsulated follicular-patterned lesions, but also have exclusion criteria that included the papillary growth pattern. ${ }^{1-4,9}$

Immunohistochemical analysis demonstrated that the adenomatous nodule-like lesion, papillary-patterned lesion with equivocal PTC-type nuclear features, and the surrounding thyroid tissue were all negative for cytokeratin 19 (clone b170, Novocastra, Newcastle upon Tyne, UK) (Fig. 2A) and BRAFV600E (clone VE1, Spring Bioscience, Pleasanton, CA, USA) (Fig 2B). The MIB-1 labeling index was estimated to be approximately $1 \%-2 \%$ in all three tested tumor areas (clone MIB-1, Dako, Glostrup, Denmark) (Fig. 2C). Subsequent mutational analysis of KRAS, NRAS, HRAS (codons 12, 13, and 61), BRAF (exon 15), and TERT promoter (C250T and C228T), as described previously, ${ }^{10}$ demonstrated that none of the three tissue samples had the BRAF mutation or the TERT promoter mutation. Interestingly, a heterozygous point mutation in exon 2 of the KRAS gene (GGT to GCT) resulting in the replacement of glycine with alanine at codon 12 (G12A), was detected in tissue samples from both the adenomatous nodule-like lesion and the papillarypatterned lesion (Fig. 2D). In contrast, the surrounding thyroid tissue was found to contain wild-type KRAS. Therefore, the nodule was found to be a clonal neoplasm but not a non-neoplastic (hyperplastic) nodule. NRAS and HRAS mutations were not detected in any of the three tumor areas.

After completing the tests described above, we speculated that this tumor (noninvasive encapsulated papillary thyroid tumor with RAS mutation) may be an example of a novel borderline tumor with papillary architecture, which does not qualify as either NIFTP or WDT-UMP under the current WHO criteria. The patient decided not to undergo additional surgery and developed no recurrence or distant metastasis during 24 months of follow-up.

Prior to June 2017, the Institutional Review Board of the Enshu Hospital waived the need for ethics approval when only archived tissue specimens were used in a retrospective manner. This study was performed in accordance with the principles of the Declaration of Helsinki. Written informed consent was obtained from the patient for the publication of this case report. 

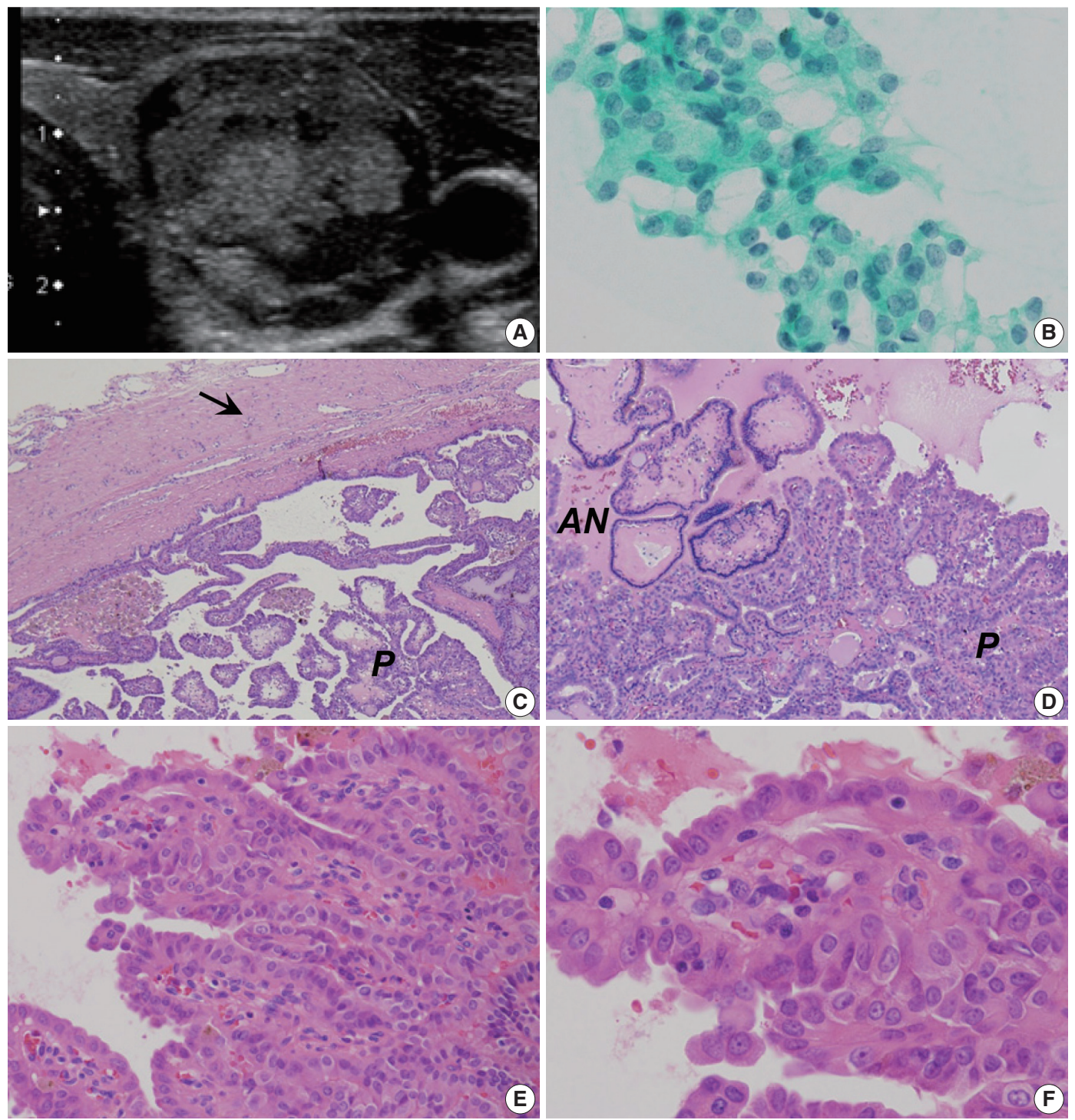

Fig. 1. Encapsulated papillary growth-patterned tumor with equivocal papillary thyroid carcinoma (PTC)-type nuclear features in a 26-year-old man (nuclear score 2 according to Nikiforov et al. ${ }^{4}$ ) (A) B-mode ultrasonography showing a hypoechoic, heterogeneous nodule with a largest diameter of $23 \mathrm{~mm}$ and a slightly jagged border. (B) Fine-needle aspiration cytology revealing a number of crowded groups of follicular cells with slight nuclear enlargement and mild nuclear overlapping (Papanicolaou stain). (C, D) The resected solid tumor showing a capsule (arrow) and two types of papillary lesions: one covered with follicular cells containing small hyperchromatic nuclei (adenomatous nodule-like lesion, AN) and the other covered with larger follicular cells having nonhyperchromatic nuclei (P). (E, F) High-power views of the second type of papillary lesion showing nuclear elongation and overlapping as well as a one- to two-fold enlargement of nuclei size with granular chromatin. Nuclear grooves are not abundant, and pseudoinclusions are absent. Chromatin characteristics of PTC-type nuclear features are insufficient.

\section{DISCUSSION}

We report an encapsulated thyroid tumor with a papillary growth pattern, nuclear score of 2 , and a KRAS mutation. The patient underwent surgery for a supposedly benign thyroid nodule that was initially diagnosed as an encapsulated PTC by a local pathologist but then as a FA with papillary hyperplasia by a second opinion consultation. Our third opinion diagnoses were high-risk indeterminate nodule (follicular neoplasm) based on FNAC, and borderline/precursor tumor based on histological findings. These discrepancies likely exist due to equivocal PTCtype nuclear changes, as NIFTP and WDT-UMP generally fall into one of the indeterminate diagnostic categories by preoperative FNAC., ${ }^{2,3}$

This study further reports that this tumor is not a $B R A F-$ mutant tumor, but rather a clonal tumor with a RAS mutation. 

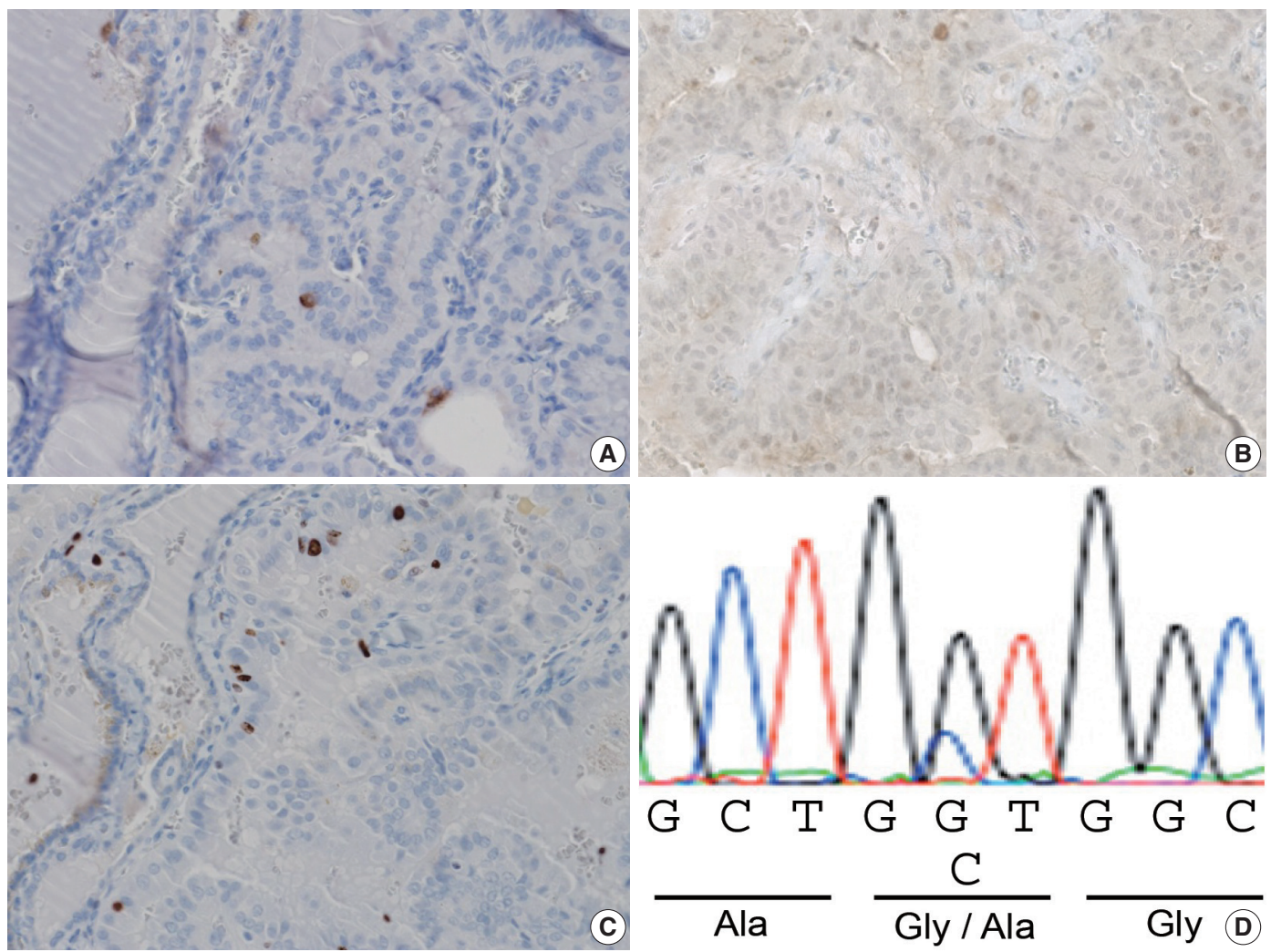

Fig. 2. Immunohistochemical and mutational analyses. (A, B) Immunohistochemical staining with cytokeratin 19 and BRAFV600E led to negative results in the cytoplasm of the tumor cells. (C) The MIB-1 labeling index was approximately 1\%-2\%. (D) Sequence analysis revealed a heterozygous point mutation of the KRAS gene in exon 2 (GGT to GCT) resulting in the substitution of alanine for glycine at codon 12 (G12A) in the tissue sample from the papillary lesion with a nuclear score of two and an adenomatous nodule-like lesion (D).

A RAS-mutant tumor became its own category when it was separated from conventional PTCs with a dominant papillary growth pattern featuring a BRAF-positive profile. ${ }^{4}$ There are PTCs in the literature that have been reported to have RAS mutations that were diagnosed as either invasive encapsulated or noninvasive encapsulated FV-PTCs. ${ }^{11-13}$ There are no detailed morphological studies of noninvasive encapsulated conventional-type PTC with a RAS mutation, and there are no available prognostic analyses. Recently, Radkay et al. ${ }^{14}$ investigated the cytopathologic features of common RAS mutation subtypes NRAS, HRAS, and KRAS. Out of 204 RAS-mutant thyroid tumors, 24 cases (11.8\%) had KRAS mutations like the one reported here. Although the surgical resection specimen demonstrated conventional PTC in two cases, detailed information on either the presence of capsulation or the degree of PTC-type nuclear changes is not available. From a review of The Cancer Genome Atlas (TCGA) study database, ${ }^{12}$ we identified four cases of PTC with KRAS mutations. Papillary growth pattern or classic type PTC was recorded in two of those cases. Case 1 (TCGA-EM$\mathrm{A} 4 \mathrm{FV}$ ) was diagnosed as invasive encapsulated PTC. The disease was stage $\mathrm{N} 0$ and the patient was disease-free 12 months postoperatively. Case 2 (TCGA-BJ-A0ZE) was diagnosed as invasive follicular variant PTC but not classic type PTC and was stage N0. The other two cases with KRAS mutations were FV-PTCs. Further studies are needed to evaluate the biological and clinical characteristics of noninvasive encapsulated tumors with a papillary growth pattern and KRAS mutations, although the frequency of such cases seems to be rare based on a survey of the current literature. ${ }^{12,14}$

Two important histological findings (encapsulated and noninvasive) and two clinical parameters (no metastasis at diagnosis and no recurrence after a long-term follow-up) are used for borderline tumor classification (i.e., HTT and NIFTP). ${ }^{1,3,4}$ Although our case demonstrated three out of four features (encapsulated, noninvasive, and no metastasis at diagnosis), the absence of recurrence after a long-term follow up is still unknown. Because of the expected low frequency of this disease, we propose that an international working group be created to confirm the extremely low grade or biologically benign nature of a possible novel subtype of borderline tumor with a papillary structure. This pro- 
posed working group study would be similar to the one conducted by the NIFTP international working group.

After the introduction of borderline thyroid tumors in the latest WHO classification, ${ }^{1-3}$ pathologists have three choices for the characterization of thyroid tumors with a follicular growth pattern: benign, borderline, and malignant. For papillary thyroid tumors, pathologists are still forced to select from either benign or malignant, which may cause significant observer variation in the diagnosis of thyroid carcinomas like the one presented in this report. Welch and Black estimated the probability of overdiagnosis of thyroid cancer to be more than $99 \% .{ }^{15}$ Therefore, the introduction of borderline tumors in papillary-patterned lesions is an urgent issue to reduce the overdiagnosis and overtreatment of indolent thyroid tumors.

The modified initial risk stratification system, published by the American Thyroid Association (ATA) in 2016, ${ }^{16}$ was incorporated into the 2017 WHO classification. ${ }^{1-3}$ In contrast, borderline tumors were still listed as "cancer" with a very low risk of structural disease recurrence $(<2 \%)$ in the 2015 ATA management guideline. ${ }^{16}$ These low-risk tumors include (1) papillary microcarcinoma, proposed as PMiT by thyroid experts; ${ }^{5}$ (2) intrathyroidal encapsulated FV-PTC (the majority of which have been downgraded to WDT-UMP or NIFTP when they are found to have no definite invasion); and (3) $\mathrm{a}<4 \mathrm{~cm}$ intrathyroidal $B R A F$ wild-type PTC. ${ }^{17,18}$ In addition to these three options, there is potential for more borderline/precursor thyroid tumors with a very low risk of disease recurrence after complete excision. Since there would be no reason for restricting borderline/precursor tumors to the follicular growth pattern, we suggest an extension of the classification for novel borderline tumors with a papillary growth pattern. Prior to the introduction of the borderline tumor category by the 2017 WHO classification, our group proposed a new prognostic classification of thyroid tumors, in which extremely low-risk tumors with both follicular and papillary architectures were grouped into borderline/precursor tumors. ${ }^{19}$ Accordingly, we propose a new term to relieve the psychological burden of patients: "noninvasive encapsulated papillary RAS-like thyroid tumor" (NEPRAS).

For providing simplified and reproducible diagnostic criteria for nuclear features, Nikiforov et al. ${ }^{4}$ developed a three-point scoring scheme, in which each category of nuclear feature is assigned a score of either 0 or 1 , yielding a summation nuclear score of $0-3$. A score of $0-1$ is diagnostic of a benign nodule, whereas a score of $2-3$ is required for diagnosing PTC-type nuclear changes. Since even delicate nuclear changes (for example, a score of two, as presented in this case) led to a categorization of PTC-type, the introduction of the borderline category in papillary growth pattern tumor classification could reduce the overdiagnosis and overtreatment of patients with indolent thyroid nodules. We would like to propose that tumors be diagnosed as FA with papillary hyperplasia, NEPRAS, and encapsulated PTC when nuclear scores are $0-1,2$, and 3 , respectively. As a consequence, a subset of FAs with papillary hyperplasia and encapsulated PTC could potentially be up- or down-graded to NEPRAS. RAS mutations were reported to correlate with significantly less developed PTC-type nuclear features and indolent clinical behaviours, ${ }^{20}$ indicating that the molecular profile of tumor cells may also play an important role in determining borderline tumors with a papillary growth pattern.

In conclusion, we report significant observer variation in a noninvasive encapsulated papillary growth-patterned tumor of more than $1 \mathrm{~cm}$ in diameter with a nuclear score of 2 and a KRAS mutation. Although further studies are required, we believe that the introduction of a borderline tumor classification for tumors with papillary structure would affect the psychological and clinical consequences related to the diagnosis of thyroid tumors.

\section{ORCID}

Kenji Ohba: https://orcid.org/0000-0001-8538-8742

Norisato Mitsutake: https:/orcid.org/0000-0002-2744-8046

Michiko Matsuse: https://orcid.org/0000-0001-6178-3342

Tatiana Rogounovitch: https://orcid.org/0000-0002-0616-3383

Kennichi Kakudo: https://orcid.org/0000-0002-0347-7264

\section{Author Contributions}

Conceptualization: KK.

Investigation: NM, MM, TR, KK.

Methodology: NM, MM, TR.

Project administration: KO.

Resources: NN, YO, YG.

Supervision: KK.

Writing—original draft: KO.

Writing—review \& editing: KO, NM, KK.

\section{Conflicts of Interest}

The authors declare that they have no potential conflicts of interest.

\section{Acknowledgments}

The authors are grateful to all the doctors who provided us with the opportunity to study the clinical course of this patient. 
The authors also thank Dr. Hiroki Mori for his valuable comments and Enago (www.enago.jp) for the English language review.

\section{REFERENCES}

1. Pappotti M, Volante M. Hyalinizing trabecular tumour. In: Lloyd RV, Osamura RY, Kloppel G, Rosai J, eds. WHO classification of tumours of endocrine organs. 4th ed. Lyon: International Agency for Research on Cancer, 2017; 73-4.

2. Chan JK, Tallini G. Tumours of uncertain malignant potential. In: Lloyd RV, Osamura RY, Kloppel G, Rosai J, eds. WHO classification of tumours of endocrine organs. 4th ed. Lyon: International Agency for Research on Cancer, 2017; 76-7.

3. Nikiforov YE, Ghossein RA, Kakudo K, et al. Non-invasive follicular thyroid neoplasm with papillary-like nuclear features. In: Lloyd RV, Osamura RY, Klöppel G, Rosai J, eds. Who classification of tumours of endocrine organs. 4th ed. Lyon: International Agency for Research on Cancer, 2017; 78-80.

4. Nikiforov YE, Seethala RR, Tallini G, et al. Nomenclature revision for encapsulated follicular variant of papillary thyroid carcinoma: a paradigm shift to reduce overtreatment of indolent tumors. JAMA Oncol 2016; 2: 1023-9.

5. Rosai J, LiVolsi VA, Sobrinho-Simoes M, Williams ED. Renaming papillary microcarcinoma of the thyroid gland: the Porto proposal. Int J Surg Pathol 2003; 11: 249-51.

6. Hirokawa M, Carney JA, Goellner JR, et al. Observer variation of encapsulated follicular lesions of the thyroid gland. Am J Surg Pathol 2002; 26: 1508-14.

7. Chan J. Strict criteria should be applied in the diagnosis of encapsulated follicular variant of papillary thyroid carcinoma. Am J Clin Pathol 2002; 117: 16-8.

8. Chan JK, Tsang WY. Endocrine malignancies that may mimic benign lesions. Semin Diagn Pathol 1995; 12: 45-63.

9. Williams ED. Guest editorial: two proposals regarding the terminology of thyroid tumors. Int J Surg Pathol 2000; 8: 181-3.

10. Mitsutake N, Fukushima T, Matsuse M, et al. BRAF(V600E) mutation is highly prevalent in thyroid carcinomas in the young population in Fukushima: a different oncogenic profile from Chernobyl. Sci
Rep 2015; 5: 16976.

11. Zhu Z, Gandhi M, Nikiforova MN, Fischer AH, Nikiforov YE. Molecular profile and clinical-pathologic features of the follicular variant of papillary thyroid carcinoma: an unusually high prevalence of ras mutations. Am J Clin Pathol 2003; 120: 71-7.

12. Cancer Genome Atlas Research Network. Integrated genomic characterization of papillary thyroid carcinoma. Cell 2014; 159: 676-90.

13. Rivera M, Ricarte-Filho J, Knauf J, et al. Molecular genotyping of papillary thyroid carcinoma follicular variant according to its histological subtypes (encapsulated vs infiltrative) reveals distinct $B R A F$ and RAS mutation patterns. Mod Pathol 2010; 23: 1191-200.

14. Radkay LA, Chiosea SI, Seethala RR, et al. Thyroid nodules with KRAS mutations are different from nodules with NRAS and HRAS mutations with regard to cytopathologic and histopathologic outcome characteristics. Cancer Cytopathol 2014; 122: 873-82.

15. Welch HG, Black WC. Overdiagnosis in cancer. J Natl Cancer Inst 2010; 102: 605-13.

16. Haugen BR, Alexander EK, Bible KC, et al. 2015 American Thyroid Association management guidelines for adult patients with thyroid nodules and differentiated thyroid cancer: the American Thyroid Association Guidelines Task Force on Thyroid Nodules and Differentiated Thyroid Cancer. Thyroid 2016; 26: 1-133.

17. Elisei R, Viola D, Torregrossa L, et al. The BRAF(V600E) mutation is an independent, poor prognostic factor for the outcome of patients with low-risk intrathyroid papillary thyroid carcinoma: singleinstitution results from a large cohort study. J Clin Endocrinol Metab 2012; 97: 4390-8.

18. Kakudo K, Bychkov A, Bai Y, Li Y, Liu Z, Jung CK. The new 4th edition World Health Organization classification for thyroid tumors, Asian perspectives. Pathol Int 2018; 68: 641-64.

19. Kakudo K, Bai Y, Katayama S, et al. Classification of follicular cell tumors of the thyroid gland: analysis involving Japanese patients from one institute. Pathol Int 2009; 59: 359-67.

20. Adeniran AJ, Zhu Z, Gandhi M, et al. Correlation between genetic alterations and microscopic features, clinical manifestations, and prognostic characteristics of thyroid papillary carcinomas. Am J Surg Pathol 2006; 30: 216-22. 